\title{
LA DEPRESIÓN EN LA INFANCIA DESDE LA PERSPECTIVA DE LAS TAXONOMÍAS EMPÍRICAS
}

\author{
CONCEPCIÓN LÓPEZ-SOLER y JOSÉ A. LÓPEZ-PINA \\ Universidad de Murcia
}

(Recibido el 20 de julio de 1997)

\begin{abstract}
En este trabajo se analiza la depresión infantil desde el modelo de taxonomías empíricas propuesto por Achenbach y Edelbrock. Se revisan algunos aspectos diferenciales en el diagnóstico de la depresión en menores y adultos. La parte empírica se centra en la aplicación de un Inventario Clínico Infantil (ICI), adaptado del Child Behavior Checklist de Achenbach y Edelbrock (1983), y de la Escala para padres de Connors. El ICI recogió información de una muestra clínica de menores, de edades comprendidas entre 6 y 12 años, En este inventario se agruparon los síntomas depresivos, siguiendo la propuesta de Clark, Lewinsohn, Hops y Seeley (1992). Se detalla la presencia de diversos síntomas depresivos en los trastornos internalizantes y la tasa de incidencia de depresión en la muestra de referencia.

Palabras clave: Depresión infantil, taxonomías empíricas, Child Behavior Checklist.

Childhood Depressive Disorder from the Empirical Taxonomies Perspective

This work aimed to analyze the Childhood depressive disorder from the Achenbach and Edelbrocks' (1983) model of empirical taxonomies. Several differential aspects of the diagnostic of depressive disorder in children and adults were reviewed. A clinical sample of 208 children between 6 and 12 years old took part in this study. Their parent filled in the Childhood Clinical Inventory (ICI) adapted both from the Achenbach and Edelbrocks' (1983) Child Behavior Checklist (CBCL) and the Conners' parent scale. The depressive symptoms were grouped according to the Clark et al.'s (1992) proposal. The presence of several depressive symptoms in the internalizing disorders and the rate of incidence of depression in the reference sample are addressed.
\end{abstract}

Key words: Childhood depression, empirical taxonomy, Child Behavior Checklist.

\section{INTRODUCCIÓN}

El tema de la depresión infantil es controvertido por varias razones. Una de ellas se refiere a la polémica acerca de si es un síndrome puro o si los síntomas básicos se manifiestan en todas las categorías psicopatológicas de la infancia (Petti, 1986). Otra razón hace referencia a si los síntomas que configuran el trastorno en la vida adulta son los mismos en edades tempranas, o si sus manifestaciones son diferentes (Kazdin, 1989). En este

Correspondencia: Concepción López-Soler. Facultad de Psicologia. Universidad de Murcia. Apdo. 4021, 30080. Murcia. Tlf.: 968 364117, Correo-e: jlpina@fcu.um.es. sentido se ha hablado de depresión enmascarada, por no ser directamente observable, en menores cuyos comportamientos se caracterizan por irritabilidad, inquietud motora y agresividad. En todo caso, lo que parece fuera de toda duda es que la depresión infantil «existe» y la polémica se centra en si se trata de un sindrome con las mismas características del que aparece en los adultos o si, por el contrario, sus manifestaciones son especificas (Shafii y Shafii, 1995).

Si revisamos los criterios diagnósticos del DSM-IV, encontramos algunas variaciones sintomatológicas para efectuar el diagnóstico de depresión en la infancia. 
Así, mientras el indicador de disforia en adultos es tristeza y/o sensación de vacío, en menores un indicador puede ser el llanto o la irritabilidad. En la anhedonia, el criterio para establecer si ha habido disminución en la capacidad para sentir placer, puede ser un comportamiento retraído y huraño. Algunos autores consideran que el retraimiento social es uno de los acompañantes permanentes de la depresión infantil (Petti, 1986), aunque tal postura no aparece en la revisión realizada por el mismo autor en 1993.

La aparente mezcla de síntomas puede estar relacionada con otro aspecto del diagnóstico clínico: la presencia de varios trastornos en los mismos menores. La comorbilidad psicopatológica es un tema relevante que se debe controlar en los estudios sobre depresión infantil, porque la presencia de síntomas que son característicos de trastornos de conducta, como la agresividad, pueden ser codeterminantes de los resultados, contaminando la generalización de los mismos. Esto es básico en las muestras clínicas por dos razones: en primer lugar porque hay una referencia más alta en caso de presentar un trastorno externalizante (hiperactividad, de conducta, oposicionista, etc.), debido a la menor manejabilidad de estos menores por parte de padres y maestros; en segundo lugar, porque es más alta la probabilidad de referencia clínica en menores que presentan varios trastornos (sesgo de Berkson). El sesgo de Berkson (1946) indica que las personas con múltiples trastornos psicológicos son referidas más fácilmente a consulta clínica que las personas con un solo trastorno. La alta frecuencia en los servicios de salud mental de personas que presentan varias alteraciones psicológicas a la vez, incrementa la estimación de comorbilidad entre trastornos en las muestras clínicas.

En los estudios sobre depresión infantil se han utilizado diversos criterios e instrumentos de evaluación, y las tasas de prevalencia en cada investigación varían en función de estas dos variables. Por un lado, si el trastorno evaluado es el distímico, la frecuencia de casos es muy superior a la encontrada respecto a la depresión mayor. En la Tabla 1 vienen especificados algunos datos referentes a la incidencia de depresión encontrada en diferentes estudios. Los criterios diagnósticos más o menos restrictivos, así como la consideración de que la sintomatología depresiva constituya o no el trastorno primario, explican las diferencias tan significativas encontradas por distintos autores. Por otro lado, las tasas de prevalencia encontradas en la depresión se ven afectadas por el instrumento de evaluación elegido. Así, la escala Children Depression Schedule suele aportar tasas más altas de prevalencia que el diagnóstico efectuado mediante criterios clínicos. En un estudio epidemiológico sobre depresión infantil publicado por Domenech y Polaino (1990), encontraron unas tasas de prevalencia del $4 \%$ y $11 \%$ para depresión mayor y distimia, respectivamente, en menores de edades comprendidas entre 8 y 10 años, mientras que en mayores de 11 años, la incidencia aumentaba hasta el $12 \%$ para la depresión mayor y al $26 \%$ en la distimia. Este incremento de sintomatología depresiva con la edad es un dato que aparece en todos los estudios, extendiéndose a todos los síndromes psicopatológicos. Costello y Angold (1995) recogen diferentes investigaciones longitudinales realizadas en poblaciones distintas. Los datos aportados permiten derivar tres ideas básicas respecto a la psicopatología infantil:

1. el incremento de problemas desde la primera infancia hasta la adolescencia.

2 . la influencia que tiene el criterio y/o instrumento de diagnóstico en las tasas de prevalencia encontradas.

3. la continuidad y permanencia de los problemas psicológicos a lo largo del ciclo vital. 
Tabla 1. Tasas de prevalencia

\begin{tabular}{lllll}
\hline Autores & Criterio & Incidencia & Edad & Muestra \\
\hline Rutter et al. (1979) & DM & $0,1 \%$ & $10-11$ & Normal \\
Albert y Beck (1975) & CDS & $40 \%, 33 \%$ & $12-16$ & Normal \\
Kashani et al. (1979) & DM & $1,9 \%$ & $15-19$ & Normal \\
& Tristeza & $17,4 \%$ & & \\
Kashani et al. (1981) & DM & $7 \%$ & & Pediátrica \\
Kashani et al. (1983) & Distimia & $2,5 \%$ & 9 & General \\
& Distimia & $\mathbf{8} \%$ & 14,16 & General \\
Kashani et al. (1987) & DM & $4,7 \%$ & 14,16 & General \\
Robbins et al. (1982) & DM & $28 \%$ & 14,18 & General \\
Kovacs (1984) & CDS & $34 \%$ & & Clínica \\
Wirt et al. (1977) & PIC & $22 \%$ & & Clínica \\
Lefkowitz et al. (1985) & Iguales & $5,2 \%$ & $6-10$ & General \\
Shaffi et al. (1992) & Tras. afec. & $34 \%$ & $>18$ & Clínica \\
\hline
\end{tabular}

Nota: $\mathrm{DM}$ = Depresión Mayor; CDS = Cuestionario de Depresión para niños; PIC = Inventario de Personalidad para niños.

Un dato significativo en la evolución de la depresión infantil es que la prevalencia es mayor en niños que en niñas, en edades tempranas, mientras que en la adolescencia, el patrón se invierte y la prevalencia es más alta en las niñas. Por otro lado Costello y Angold (1995), también informan que hay un patrón de continuidad y otro de cambio en psicopatología infantil. El primero, de continuidad, se refiere a la permanencia e incremento de indicadores psicopatológicos hasta la adolescencia. El segundo, de cambio, indica la modificación del tipo concreto de trastorno, ya que en los niños se incrementa la probabilidad de presentar un problema de tipo externalizante en la adolescencia, cuando a los 10 años se ha tenido un problema internalizante o externalizante.

En la evaluación de los comportamientos psicopatológicos en la infancia y adolescencia se utilizan una serie de escalas estructuradas entre las que destacan la DICA (Diagnostic Interview for Children and Adolescent) de Herjanic y su grupo (Herjanic, Herjanic, Brown y Wheatt, 1975; Herjanic y Reich, 1983), la CAS (Child Assessment Schedule), de Hodges, Kline, Fitch, Mckrew y Cytrym
(1981), la DISC (Diagnostic Interview Schedule for Children) de Costello et al. (1982) y la CBCL (Child Behavior Checklist) de Achenbach y Edelbrock (1983). Todas estas escalas permiten establecer diagnósticos de una amplia gama de trastornos infantiles, permitiendo un análisis de comorbilidad.

La CBCL es un inventario o listado de conductas, ampliamente utilizada en el estudio de taxonomías empíricas en psicopatología infantil. Consta de 113 ítems de conductas patológicas, además de escalas de habilidades sociales. La factorización de los ítems ha determinado un conjunto de síndromes empíricos que se sintetizan en la Tabla 2. En esa misma tabla aparecen los hallados en una investigación realizada por nuestro grupo, en una muestra clínica, con una adaptación de la escala de Achenbach y de la escala de hiperactividad de Conners, que hemos denominado Inventario Clínico Infantil (ICI). La factorización de la escala proporciona alrededor de 10 factores primarios, denominados de banda estrecha, cuya factorización posterior permite obtener dos grandes síndromes empíricos de banda ancha, denominados internalizante y externalizante. El Inventario Clínico 
Infantil consta de 96 ítems que exploran diversos tipos de comportamientos desadaptados en menores. Se ha trabajado con él en una muestra clínica de niños y niñas de la consulta de Psicología Clínica del Hospital Virgen de la Arrixaca de Murcia. Los resultados acerca de los síndromes empíricos derivados del inventario muestra alrededor de ocho posibles tipos de alteraciones psicopatológicas en menores con problemas. En el primer trabajo realizado (López Soler et al., 1995) se obtuvieron siete factores, tres internalizantes y cuatro externalizantes. Posteriormente (López Soler, en prensa), mediante la aplicación del scree-plot se decidió seleccionar ocho síndromes empíricos.

En las Tablas 3 y 4 aparecen los síndrome empíricos obtenidos en la factorización del ICI en base a los síntomas listados. Como se puede observar, el factor de depresión no coincide con el hallado por Achenbach y Edelbrock (1983), ya que hemos encontrado un factor mixto de ansiedad-depresión. Por otra parte nuestros resultados coinciden con los de Lemos, Fidalgo, Calvo y Menéndez (1992), que también hallaron este factor mixto en un estudio realizado en adolescentes sin referencia clínica empleando la YSR (Youth Self Reports) de Achenbach (1985), que es una escala semejante a la CBCL, adaptada para autoinforme.

\section{OBJETIVOS}

La meta de esta investigación es conocer la tasa de incidencia de sintomatología depresiva en una muestra de población clínica infantil, así como la tasa de incidencia de trastornos internalizantes, en la misma.

Tabla 2. Síndromes empíricos internalizantes y externalizantes en niños y niñas en las escalas CBCL e ICI

\begin{tabular}{|c|c|c|}
\hline INTERNALIZANTES & CBCL & ICI \\
\hline Niñas & $\begin{array}{l}\text { Depresión } \\
\text { Retraimiento Social } \\
\text { Quejas somáticas } \\
\text { Esquizoide/obsesivo }\end{array}$ & $\begin{array}{l}\text { Ansiedad/Obsesión } \\
\text { Reservas/Quejas somát. } \\
\text { Suspicacia }\end{array}$ \\
\hline Niños & $\begin{array}{l}\text { Depresión } \\
\text { Poca comunicación } \\
\text { Quejas somáticas } \\
\text { Esquizoide }\end{array}$ & $\begin{array}{l}\text { Ansiedad/Obsesión } \\
\text { Quejas somáticas } \\
\text { Esquizoide }\end{array}$ \\
\hline \multicolumn{3}{|l|}{ EXTERNALIZANTES } \\
\hline Niñas & $\begin{array}{l}\text { Depresión } \\
\text { Hiperactividad } \\
\text { Agresividad } \\
\text { Delincuencia } \\
\text { Crueldad }\end{array}$ & $\begin{array}{l}\text { Ansiedad/Obsesión } \\
\text { Problemas de Atención } \\
\text { Agresividad verb/hp } \\
\text { Desafío }\end{array}$ \\
\hline Niños & $\begin{array}{l}\text { Depresión } \\
\text { Hiperactividad } \\
\text { Agresividad } \\
\text { Delincuencia }\end{array}$ & $\begin{array}{l}\text { Ansiedad/Obsesión } \\
\text { Problemas de Atención } \\
\text { Agresividad } \\
\text { Robos } \\
\text { Autoagresión }(\mathrm{m})\end{array}$ \\
\hline
\end{tabular}


Tabla 3. Síndromes empíricos externalizantes en niños. Inventario Clínico Infantil (ICI)

\begin{tabular}{llll}
\hline FACTOR I & FACTOR II & FACTOR V & FACTOR VII \\
\hline Ira & Dif. Aprendizaje & Autoagresión & Robos \\
Desafia & Cond. Infantil & Mirada fija ojos & Amable \\
Maldice & Impulsivo & Rehusar hablar & Siente no le quieren \\
Discute & Destruye cosas & Hablar de matarse & \\
Burlas & Distraído & Preocupado sexo & \\
Quejas & Dif. Instrucciones & & \\
Agrede físicamente & Destruye c. Propias & & \\
Se burla & Confusión & & \\
Chilla & Nervioso, tenso & & \\
Cond. e ideas extr. & Prob. Hablar & & \\
Irritable & & & \\
Cond. Explosiva & & & \\
Amenaza & & & \\
Crueldad & & & \\
Peleón & & & \\
Cambios de humor & & & \\
Agitación nerviosa & & & \\
Frustable & & FACTOR VI & \\
Sospecha & & Vómitos, nauseas & \\
FACTOR III & & Agitación nerviosa & \\
Triste, deprimido & Inseguridad & Dolor estómago & \\
Hace ruido extra & Temor equivocarse & \\
Reservado & Vergüenza & \\
Oye cosas no están & Vulnerable crítica & Nervioso, tenso & \\
Cruel, abusa & Inferioridad & & \\
Falla en tareas & Preocupado & & \\
Apático & Solitario & \\
Obstinado, irritable & Temor hacer/pensar & \\
Repite acciones & Culpabilidad & \\
Llora mucho & & & \\
Ve cosas no están & & & \\
\hline
\end{tabular}

Tabla 4. Síndromes empíricos externalizantes en niñas. Inventario Clínico Infantil (ICI)

\begin{tabular}{|c|c|c|c|}
\hline FACTOR I & FACTOR II & FACTOR III & FACTOR VII \\
\hline $\begin{array}{l}\text { Chilla } \\
\text { Discute } \\
\text { Habla mucho } \\
\text { Irritable } \\
\text { Nervioso } \\
\text { Burlas } \\
\text { Habla alto } \\
\text { Habla f. turno } \\
\text { Participa pelea } \\
\text { Agitación } \\
\text { Impulsividad } \\
\text { Se pellizca } \\
\text { Burlas a otros } \\
\text { Cond. explosiva } \\
\text { Se queja }\end{array}$ & $\begin{array}{l}\text { Dif. aprendizaje } \\
\text { Cond. infantil } \\
\text { Distracción } \\
\text { Problema hablar } \\
\text { Falla tareas } \\
\text { Dif. instrucciones } \\
\text { Confusión } \\
\text { Mov. nerviosos } \\
\text { Lenguaje obsceno }\end{array}$ & $\begin{array}{l}\text { Sueña despierta } \\
\text { Agresión física } \\
\text { Se burla } \\
\text { Impulsividad } \\
\text { Destruye cosas } \\
\text { No termina tareas } \\
\text { Dominancia } \\
\text { V. problemáticos } \\
\text { Vergüenza }\end{array}$ & $\begin{array}{l}\text { Desafía } \\
\text { Vulnerable crítica } \\
\text { Amable } \\
\text { Dolor cabeza }\end{array}$ \\
\hline FACTOR IV & FACTOR V & FACTOR VI & \\
\hline $\begin{array}{l}\text { Pequeños hurtos } \\
\text { Habla de matarse } \\
\text { Solitaria } \\
\text { Preocupación sexo } \\
\text { Dolor estómago } \\
\text { Culpabilidad } \\
\text { Ve cosas no están }\end{array}$ & $\begin{array}{l}\text { Sospecha } \\
\text { Tensión, nervios } \\
\text { Confusión } \\
\text { Ruidos extraños } \\
\text { Ira } \\
\text { Alardea, payasea } \\
\text { Habla matarse } \\
\text { Agitación }\end{array}$ & $\begin{array}{l}\text { Miedo, ansiedad } \\
\text { Temor equivocarse } \\
\text { Perfeccionismo } \\
\text { Repite acciones } \\
\text { Obsesiones } \\
\text { Preocupación } \\
\text { Inseguridad }\end{array}$ & \\
\hline
\end{tabular}




\section{METODOLOGÍA}

\section{Participantes}

Hemos trabajado con un grupo de niños y niñas de la consulta de Psicología Clínica de la Sección de Psiquiatría, del Hospital Universitario Virgen de la Arrixaca de Murcia. Todos los menores procedían de las interconsultas efectuadas principalmente por Psiquiatría, Neuropediatría, Pediatría, Cirugía Infantil y Neurocirugía, y habían sido remitidos a nuestra consulta por presentar indicadores de desajuste psicológico.

La muestra consta de 122 niños y 60 niñas de edades comprendidas entre los 6 y 11 años de edad. Ninguno de ellos padecía retraso mental, todos seguían régimen de escolaridad normal y pertenecen a familias sin handicaps sociales significativos, de nivel económico y cultural medio.

\section{Instrumento de medida}

De todos los menores se obtuvo información con respecto a una serie de características comportamentales con posible significado psicopatológico. El instrumento a través del cual se consiguió esta información fue el Inventario Clínico Infantil (ICI) (López Soler, 1986), que es una adaptación del CBCL de Achenbach y Edelbrock, y del Cuestionario para Padres de Conners. El ICI consta de 96 ítems, y en un estudio de validación previo (López Soler, en prensa) se obtuvo un coeficiente de fiabilidad (alfa) de 0,96. El inventario se informa por los padres, normalmente la madre, ya que suele ser la persona que acompaña al menor en la consulta, en la primera visita, con el fin de evitar que en la información recogida influya la intervención terapéutica. Cada ítem es calificado mediante una escala de cuatro niveles, en función de la frecuen- cia de aparición de la conducta concreta en los últimos meses (nunca, algunas veces, a menudo y siempre).

\section{Tratamiento estadístico}

Los datos obtenidos en los inventarios se analizaron factorialmente y son objeto de otro trabajo (López Soler, en prensa). Se extrajeron siete factores cuya composición y denominación aparece en las Tablas 3 y 4.

Para saber el número de casos de nuestra muestra que presentan un trastorno depresivo, listamos los síntomas que configuran clínicamente dicho trastorno, y que aparecen en el trastorno de ansiedaddepresión o en alguno de los trastornos internalizantes, y el criterio seguido se basa en las recomendaciones de Clark, Lewinshon, Hops y Seeley (1992) que han propuesto una escala de depresión a partir de la CBCL (CBCL-D). La utilización de este criterio se debe a que en nuestro estudio no aparece un factor de "depresión puro» en este rango de edad, ya que no aparecen parte de los síntomas básicos, como ideas de suicidio, y se configura con otros de ansiedad. La escala ICI-Depresión está formada por los siguientes ítems: tristeza, apatía, llanto, reserva, culpabilidad, soledad, preocupación, vulnerabilidad a la crítica, inferioridad, vergüenza, temor a equivocarse, inseguridad y tensión.

\section{RESULTADOS}

\section{Depresión-distimia}

A) En lo referente al primer objetivo de la investigación, tanto en niños como en niñas los ítems que configuran el trastorno depresivo y distímico, se distribuyen en los tres factores internalizantes; en niños encontramos tristeza, reserva, 
apatía y llantos en el factor pensamiento esquizoide; y nervioso y tenso en el de quejas somáticas. No obstante en este caso el factor depresión-ansiedad incluye la mayoría de los síntomas clínicos de la depresión. Por el contrario en las niñas, aparecen menos síntomas específicos de depresión en los diferentes factores, y el factor específico de ansiedaddepresión muestra menos ítems de distimia e incluye los síntomas típicos del trastorno obsesivo-compulsivo (perfeccionismo, repite acciones y obsesiones).

Por lo que se refiere al numero de casos que presentan los síntomas especificados en el ICI-D, en la Tabla 5 se muestran los porcentajes de niños y niñas que sufren depresión: un $17,21 \%$ en los primeros y un $15 \%$ en las segundas; un contraste de significación de proporciones muestra que las diferencias entre ambos grupos no fueron significativas $(z=0,38$, $p>0,05$ ).

B) Respecto a la presencia de algún trastorno internalizante, diagnosticado a partir del ICI, siguiendo el criterio antes expuesto, hemos hallado un $30,56 \%$ de casos con problemas de tipo quejas somáticas, problemas de pensamiento (esquizoide o suspicacia) y/o ansiedad-depresión. Su distribución por sexos es la siguiente: un $32,79 \%$ en niños y un $28,33 \%$ en niñas (ver Tabla 5), no encontrándose diferencias significativas entre ambos grupos $(z=0,61, p>0,05)$.

Hemos obtenido también la tasa de trastornos externalizantes y aunque en

Tabla 5. Síndromes internalizantes y externalizantes

\begin{tabular}{lccc}
\hline & Niños & Niñas & Instrumento \\
\hline Internalizante & $\mathbf{3 2 , 7 9 \%}$ & $\mathbf{2 8 , 3 3 \%}$ & ICI \\
Externalizante & $\mathbf{2 7 , 8 7 \%}$ & $\mathbf{1 8 , 3 3 \%}$ & ICI \\
Depresión & $17,21 \%$ & $\mathbf{1 5 , 0 0 \%}$ & ICI-D \\
\hline
\end{tabular}

los dos subgrupos el porcentaje de casos disminuye, sigue siendo considerablemente más alto en niños $(27,87 \%$ y $18,33 \%$ ), aunque no se encontraron diferencias significativas entre ambos $(z=$ $1,40, p>0,05)$.

\section{DISCUSIÓN}

En primer lugar, nuestros resultados coinciden con los hallazgos generales acerca de la mayor tasa de índices psicopatológicos en niños que en niñas, y tanto en los internalizantes como en externalizantes, siendo respecto a este último más marcadas las diferencias entre ambos sexos. Tanto en niños como en niñas hay un alto porcentaje de casos con problemas de tipo externalizante, siendo de nuevo la tasa más elevada la de los niños.

En segundo lugar, y por lo que se refiere al rango de edad estudiado (6-11 años), la depresión no aparece como un factor integrado, por el contrario encontramos un factor mixto de ansiedad-depresión y «rasgos depresivos" en los factores de quejas somáticas y problemas de pensamiento. Si bien en los niños encontramos dos peculiaridades: primero, que ese factor específico está mejor configurado que en las niñas, dato que coincide con los hallazgos en adolescentes de Lemos et al. (1992), siendo más equivalente a la caracterización clínica de la depresión; y segundo, que el factor de problemas de pensamiento de tipo esquizoide que hemos detectado, presenta cuatro síntomas típicos de la depresión: tristeza, reserva, apatía y llanto. La presencia de reserva y apatía en el trastorno esquizoide, y de "solitario" en el de depresiónansiedad, así como la alta presencia de otros problemas internalizantes, podría explicar la postura de algunos autores, como Petti (1986), de considerar que el retraimiento es compañero inseparable de la depresión. 
Finalmente parece interesante resaltar el hecho de dos síntomas prototípicos del cuadro, tristeza y apatía, aparezcan configurando el factor de "problemas de pensamiento de tipo esquizoide» en los niños. No obstante, y puesto que se trata de un estudio piloto realizado sobre una muestra pequeña, creemos que no es prudente generalizar a otras muestras clínicas, por lo que conviene realizar un estudio con metodología similar en un grupo representativo de la población de referencia, tarea que estamos llevando en colaboración con profesionales de centros de Salud Mental de la Región de Murcia.

\section{REFERENCIAS BIBLIOGRÁFICAS}

Achenback, T.M. (1985). Assessment and Taxonomy of Child and Adolescent Psychopathology. L/A: Sage Publications.

Achenbach, T.M. y Edelbrock, C. (1983). Manual for the Child Behavior Checklist and Revised Child Behavior Profile. Burlington, Vermont: University of Vermont. Clark, G.N., Lewinsohn, P.M., Hops, H. y Seeley, J.R. (1992). A Self- and Parents-Report Measure of Adolescent Depression: the Child Behavior Cheklist Depression Scale (CBCL-D). Behavioral Assessment, 14, 443463.

Costello, A., Edelbrook, C., Kalas, R., Kessler, M. y Klaric, S.H. (1982). NIMH DISC-II (Working Draft). Washington, D.C.: NIHM. Costello, E.J. y Angold, A. (1995). Developmental Epidemiology. En D. Chichetti y D.J. Cohen (Eds.), Developmental Psychopathology: Theory and Methods. New York: Wiley Interscience.

Domenech Llaberia, E. y Polaino-Lorente, A. (1990). Epidemiología de la Depresión Infantil. Barcelona: Expaxs.
Herjanic, B., Herjanic, M., Brown, F. y Wheatt, J. (1975). Are children reliable reporters? Journal of Abnormal Child Psychology, 3, 41-48.

Herjanic, B. y Reich, W. (1982). Development of a structured psychiatric interview for children: Agreement between parent on individual symptoms. Journal of Abnormal Child Psychology, 10, 307-324.

Hodges, K., Kline, J., Fitch, P., Mckrew, D. y Cytrym, L. (1981). The child assessment schedule: A diagnostic interview for research and clinical use. JSAS Catalog of Selected Documents in Psychology, 11, 56.

Kazdin, A.E. (1989). Evaluation of the pleasure scale in the assessment of anhadonia in children. J. Am. Acad. Child and Adolesc. Psychiatry, 28, 364-372.

Lemos, S., Fidalgo, A.M., Calvo, P. y Menéndez, P. (1992). Estructura Factorial de la prueba YSR y su utilidad en Psicopatología Infanto-Juvenil. Análisis y Modificación de Conducta, 18, 884-905.

López Soler, C. (1986). Inventario Clínico Infantil (ICI). Manuscrito no publicado.

López Soler, C. (en prensa). Estructura Factorial del Inventario Clínico Infantil (ICI, Adaptación del CBCL), en Niños y Niñas con Problemas de Conducta y Emocionales. Estrés y Ansiedad.

López Soler, C., García, C., Murcia, L., Martín, C., Cortegano, C., López, Y. y López, E. (1995). Problemas Psicopatológicos en una muestra clínica de Niños y Niñas: Taxonomías Empíricas. Anales de Psicología, 11, 129-141.

Petti (1986). Retraimiento y Depresión. En Hersen y Ollendick (Ed.), Psicopatología Infantil. Barcelona: Martínez-Roca.

Petti (1993). Depresión. En Hersen y Ollendick, Psicopatología Infantil. Barcelona: Martínez-Roca.

Shafii, M. y Shafii, S.L. (1995). Depresión en Niños y Adolescentes: Clínica, Evaluación y Tratamiento. Barcelona: Martínez-Roca. 